\title{
Transient neonatal tricuspid regurgitation: a Doppler echocardiographic study of three cases
}

\author{
MARC GEWILLIG, MONIQUE DUMOULIN, LUCAS G VAN DER HAUWAERT \\ From the Section of Paediatric Cardiology, Department of Paediatrics, Gasthuisberg University Hospital, \\ Leuven, Belgium
}

SUMMARY Three patients with normal hearts and no pulmonary abnormality had neonatal tricuspid regurgitation causing cardiorespiratory distress and cyanosis. The signs of tricuspid regurgitation resolved over a few weeks. In the acute phase echocardiography showed gross dilatation of the right atrium and ventricle. The interatrial septum bulged into the left atrium during the whole cardiac cycle. Doppler echocardiography showed clinically significant tricuspid regurgitation, a right to left shunt through the foramen ovale, reduced flow through the pulmonary valve, and in two patients ductal flow into the pulmonary artery. In one patient tricuspid regurgitation was so great that it impeded the opening of the pulmonary valve and produced functional "atresia" of the pulmonary valve. The presence of regurgitant blood flow through the pulmonary valve showed that the "atresia" was functional rather than organic. Doppler echocardiographic study is useful in distinguishing functional neonatal tricuspid regurgitation from structural abnormality of the tricuspid valve.

The clinical diagnosis of transient neonatal regurgitation is difficult. ${ }^{1-3}$ In the past the diagnosis was confirmed by cardiac catheterisation and angiography. ${ }^{3}$ It is likely that only patients who were severely affected needed invasive investigation. ${ }^{46}$ Doppler echocardiography permits an accurate diagnosis of atrioventricular valve regurgitation. Our observations in three infants illustrate the clinical spectrum of transient neonatal tricuspid regurgitation and show the value of Doppler echocardiography in its assessment.

\section{Patients and methods}

From August 1985 to the end of February 1986 seven neonates with clinical, radiographic, and echocardiographic evidence of important tricuspid regurgitation and an otherwise structurally normal heart were seen. Three of these patients had severe pulmonary disease: two had hyaline membrane disease and one had pneumonitis caused by meconium aspiration. One neonate presented with congestive heart failure and a hyperkinetic circulation caused by

Requests for reprints to Dr Lucas G Van der Hauwaert, Section of Paediatric Cardiology, Gasthuisberg University Hospital, 3000 Leuven, Belgium.

Accepted for publication 23 May 1988 a large cerebral arteriovenous fistula. In the remaining three patients we found no underlying abnormality. In these three patients all signs of tricuspid regurgitation resolved over a few weeks or months. They form the basis of this report.

Doppler echocardiographic studies were performed with commercial units. We did not measure the size of the atrium as there are no universally accepted normal reference standards. Only gross disproportions between the atria were noted. Bulging of the interatrial septum into the left atrium was assessed throughout the cardiac cycle. Dilatation of the right ventricle was measured by comparing the right and left ventricular end diastolic dimensions in the parasternal short axis view. Tricuspid regurgitation was assessed according to the classification proposed by Miyatake et al. ${ }^{7}$

The pressure drop across the tricuspid valve during systole was estimated from the maximum flow velocity recorded by continuous wave Doppler and the simplified Bernouilli equation $\left(p=4 v^{2}\right.$ where $p$ $=$ peak pressure gradient in $\mathrm{mm} \mathbf{H g}$ and $\mathrm{v}=$ maximal flow velocity in $\mathrm{m} / \mathrm{s}$ ).

CASE 1

A full term male infant (birth weight $3700 \mathrm{~g}$ ) was born after an uneventful pregnancy and delivery. The one and five minute Apgar scores were 6 and 8. 
Slight cyanosis, present at birth, persisted with the infant breathing $\mathbf{4 0} \%$ oxygen. A heart murmur was noted at age 8 hours. Because of his increasing tachypnoea and grunting, an endotracheal tube was installed and positive airway pressure ventilation was started. When he was two days old he was transferred to our hospital. There was mild cyanosis and the liver was palpable below the costal margin. Auscultation of the lungs was normal. A grade 4/6 high pitched pansystolic murmur was best heard at the lower left sternal border. The chest $x$ ray showed mild cardiomegaly with a cardiothoracic ratio of 0.57 and normal hilar markings. Right atrial enlargement and ST depresssion with negative $T$ waves from V3R to V6 were noted on echocardiography.

Cross sectional echocardiograms showed a structurally normal heart but pronounced dilatation of the right atrium and right ventricle. The interatrial septum bowed towards the left atrium throughout most of the cardiac cycle, suggesting a large pressure difference. A ductus arteriosus was visualised. On $M$ mode echocardiograms the ratio of the right ventricle to the left ventricle at end diastole was 0.86 . The next day the condition had improved and the patient could be extubated. Cross sectional echocardiography showed that the right ventricle had become smaller and that there was a straight interatrial septum. Pulsed Doppler showed a grade 3 pansystolic tricuspid regurgitant jet halfway down the right atrium. Because the maximum flow velocity was $3.0 \mathrm{~m} / \mathrm{s}$ we estimated that the systolic right ventricular-right atrial gradient was $36 \mathrm{~mm} \mathrm{Hg}$. All other flow velocities and flow patterns were normal except for turbulence in the pulmonary artery caused by ductal flow. The cardiac murmur disappeared completely over the next week. Follow up examinations at one week and two months were within normal limits.

\section{CASE 2}

This male infant was delivered by caesarean section because of fetopelvic disproportion. The mother had been injecting 8 units of insulin daily during the second half of pregnancy. At birth the patient appeared to be normal but a few hours later he became cyanotic and tachypnoeic. Because a heart murmur was heard echocardiography was performed; this led to the tentative diagnosis of "hypoplastic left heart syndrome". When he was two days old he was transferred to our hospital. On examination tachypnoea, mild cyanosis, and gross hepatomegaly were noted. A grade 2/6 systolic murmur and a gallop rhythm were heard at the lower left sternal border. The electrocardiogram was within normal limits. The chest $x$ ray showed considerable cardiomegaly (cardiothoracic ratio 0.75 ) and pulmonary hypoperfusion.

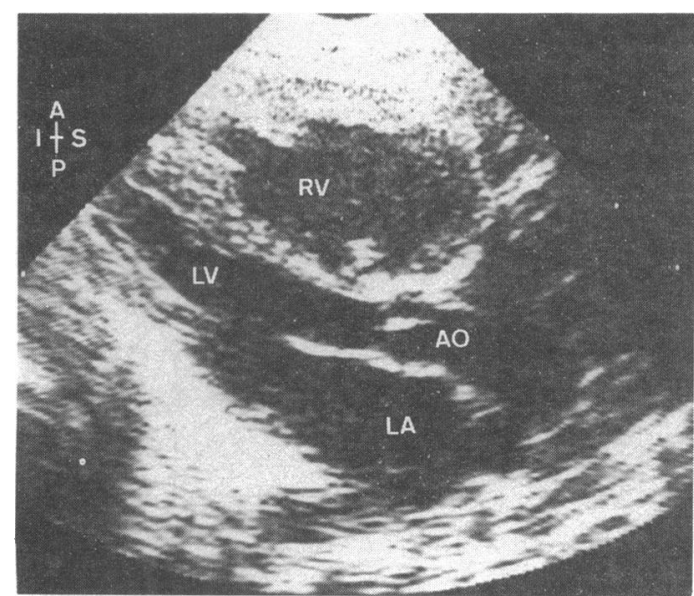

Fig 1 Parasternal long axis view in patient 2 showing a normal size left heart and a considerably dilated right ventricle. This gross disproportion between the ventricles had led to the erroneous diagnosis of hypoplastic left heart. $A O$, ascending aorta; $L A$, left atrium; $L V$, left ventricle; $R V$, right ventricle; $A$, anterior; $I$, inferior; $P$, posterior; $S$, superior.

Cross sectional echocardiography (fig 1) showed that the dimensions of the left heart were normal but that the right atrium, right ventricle, and pulmonary trunk were grossly dilated. On $\mathbf{M}$ mode echocardiograms the ratio of the right ventricle to the left ventricle was 1.0 at end diastole. There was no evidence of a ductus arteriosus. Pulsed Doppler echocardiography showed considerable pansystolic retrograde turbulent flow into the right atrium, corresponding to grade 3 tricuspid regurgitation. A maximum flow velocity of $3.1 \mathrm{~m} / \mathrm{s}$ indicated a systolic pressure drop of $38 \mathrm{~mm} \mathrm{Hg}$ from the right ventricle to the right atrium. A small regurgitant jet was recorded just proximal to the pulmonary valve. During the next few days the patient's condition improved rapidly and signs of heart failure disappeared. When he was one week old the systolic murmur and gallop rhythm were no longer audible. On the echocardiogram only borderline enlargement of the right atrium and ventricle persisted. Doppler studies at follow up were within normal limits.

\section{CASE 3}

A female infant (birth weight $2900 \mathrm{~g}$ ) was born after an uncomplicated pregnancy. The mother had taken no medication. The five minute Apgar score was 10. The same day the baby was referred because of suspected congenital heart disease. On physical examination mild cyanosis and tachypnoea were noted. The precordial impulse was active and diffuse. The liver was palpable $4 \mathrm{~cm}$ below the costal margin. The second sound was single. A grade $2 / 6$ systolic 


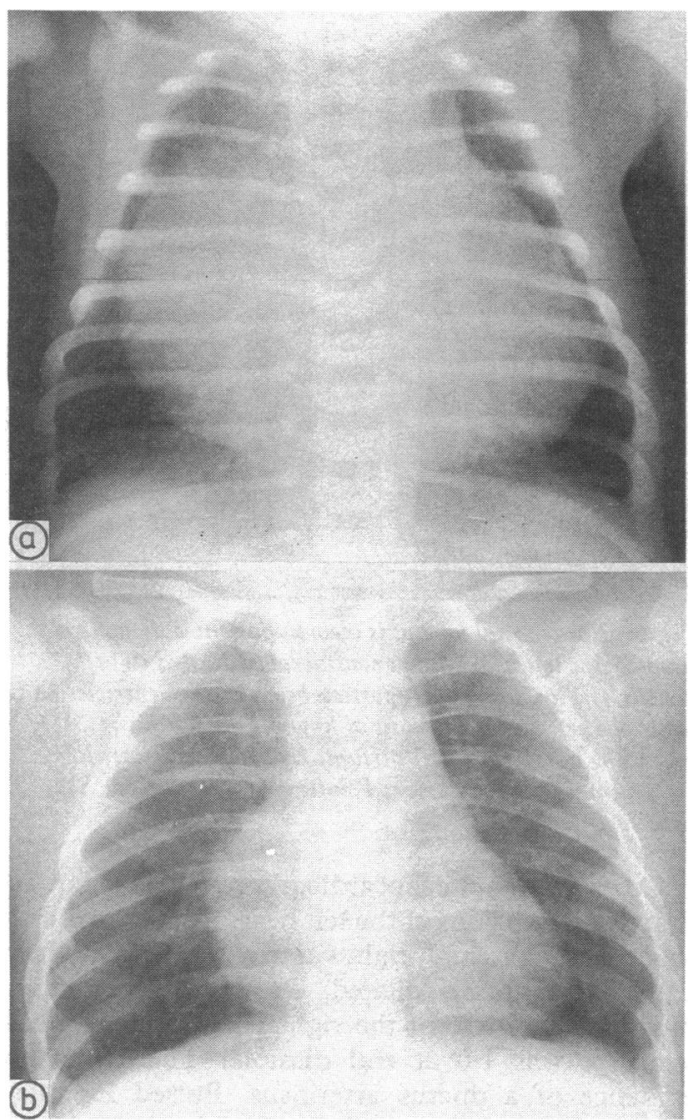

Fig 2 The chest $x$ ray when patient 3 was one day old (a) showing massive cardiomegaly caused by dilatation of the right atrium and ventricle. At age 3 months (b) only slight right atrial dilatation persisted.

murmur and a diastolic rumble were heard at the lower left sternal border. The chest $x$ ray showed massive cardiomegaly (cardiothoracic ratio 0.9 ) (fig 2a). Right atrial enlargement, pronounced right ventricular hypertrophy with deep $Q$ waves in V3R, and ST changes were noted on the electrocardiogram.

Cross sectional echocardiography showed gross dilatation of the right atrium and right ventricle (fig 3). The interatrial septum bulged into the left atrium throughout the cardiac cycle. The right ventricular outflow tract and pulmonary trunk were well developed. The pulmonary valve was thickened and immobile. The tricuspid valve was echodense but moved well. There was no apical displacement of the annulus. The ductus arteriosus was visualised and retrograde flow was seen in the pulmonary artery. The inferior angle between the ductus and the descending thoracic aorta was obtuse. $M$ mode

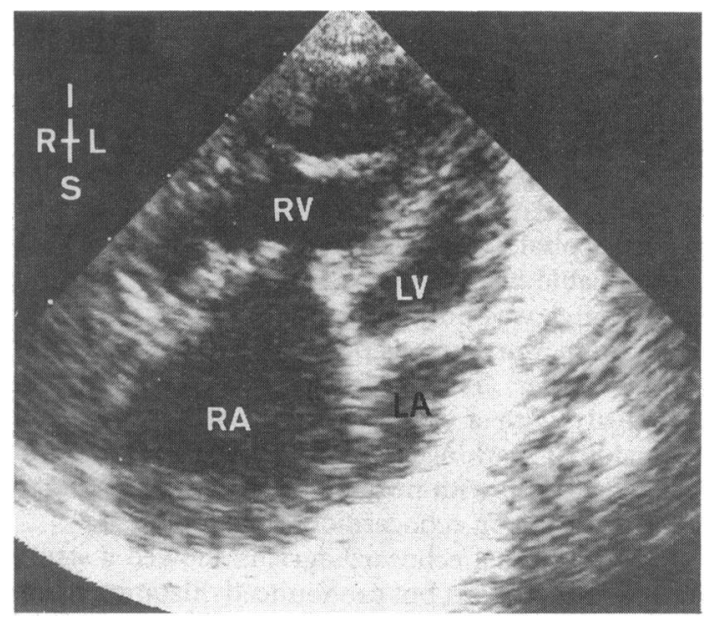

Fig 3 The apical four chamber view in patient 3 showing considerable dilatation of the right atrium and right ventricle and bulging of the atrial septum into the left atrium. The apex of the heart was found to consist mainly of the right ventricle even when the transducer was moved as far as possible to the left. $L A$, left atrium; $L V$, left ventricle; $R A$, right atrium; $R V$, right ventricle; $I$, inferior; $L$, left; $R$, right; $S$, superior.

echocardiograms showed a right ventricular/left ventricular ratio of $1 \cdot 2$. Doppler echocardiography showed no forward flow across the pulmonary valve. A pulmonary regurgitant jet was identified in the right ventricular outflow tract; this finding excluded pulmonary atresia but not severe pulmonary stenosis. The continuous Doppler beam could not be aligned satisfactorily with this regurgitant jet. The maximum recorded speed of $2.9 \mathrm{~m} / \mathrm{s}$ was consistent with a diastolic gradient across the pulmonary valve of at least $33 \mathrm{~mm} \mathrm{Hg}$. A pansystolic tricuspid regurgitant flow was recorded in the entire right atrium and in the hepatic veins. The maximal velocity on a satisfactory signal was $2.9 \mathrm{~m} / \mathrm{s}$ corresponding to a pressure drop of $33 \mathrm{~mm} \mathrm{Hg}$ from the right ventricle to the right atrium during systole (fig 4). These findings suggested that pressure in the right ventricle remained below that in the pulmonary trunk throughout the cardiac cycle.

Over a period of three months the clinical condition improved steadily - the respiratory rate became normal and the hepatomegaly and cyanosis disappeared. Physical examination when the patient was five weeks old showed a soft grade $2 / 6$ systolic murmur at the fourth left intercostal space and a normally split second sound. Cross sectional echocardiography showed a striking reduction in the dimensions of the right heart; the right atrium was still slightly dilated but the right ventricle was within 


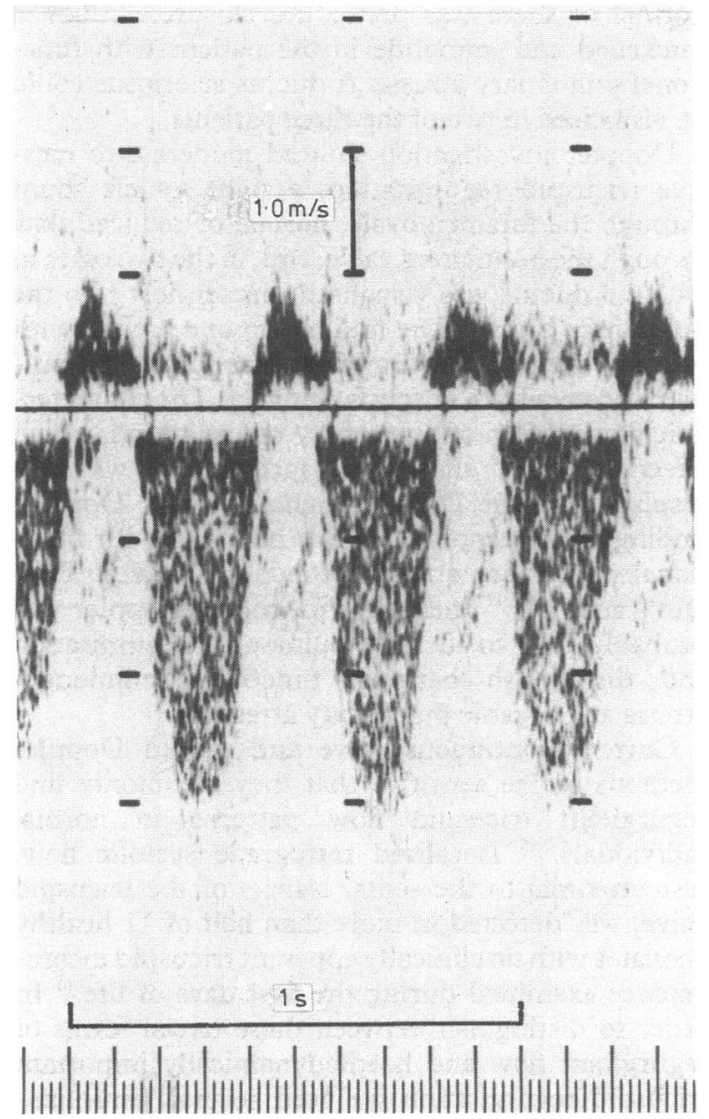

Fig 4 Continuous wave Doppler tracing from near the apex in patient 3. The maximum flow velocity of the pansystolic tricuspid regurgitant jet $(2.9 \mathrm{~m} / \mathrm{s})$ suggests a gradient of $33 \mathrm{mom} \mathrm{Hg}$ from the right ventricle to the right atrium during systole.

normal limits. The appearance and motion of the tricuspid and pulmonary valves were normal. Doppler echocardiography showed a grade 1 tricuspid regurgitant jet and trivial pulmonary regurgitation. Clinical, echocardiographic, and Doppler examinations at three and six months were within normal limits. The chest $x$ ray showed slight dilatation of the right atrium (fig 2b).

\section{Discussion}

Various lesions can cause severe tricuspid regurgitation in the neonatal period. Primary anomalies of the tricuspid valve include Ebstein's malformation, tricuspid valve dysplasia, abnormal attachment of the chordae and papillary muscles, isolated clefts, isolated deficiency of one or more leaflets, unguarded tricuspid orifice, reduplication of the tricuspid orifice, and atrioventricular septal defect. ${ }^{58}$ Tricuspid regurgitation may also be secondary to severe pulmonary hypertension or obstruction of the right ventricular outflow tract with an intact ventricular septum. ${ }^{9}$ Finally, there is a transient form of isolated tricuspid regurgitation that does not have an obvious anatomical and haemodynamic basis. ${ }^{1-3}$ It has been suggested that sudden perinatal constriction of the ductus arteriosus forces the right ventricle to pump the entire systemic venous return into the high resistance pulmonary circuit. This might result in right ventricular dilatation and tricuspid regurgitation that would resolve with the reduction of pulmonary resistance and pressure during the first days of life. ${ }^{2610}$ Others speculate that perinatal hypoxia leads to myocardial ischaemia and dysfunction of the papillary muscles of the tricuspid valve. ${ }^{411}$

The clinical syndrome of transient tricuspid regurgitation in the neonate is variable and depends on the degree of valve dysfunction. In milder forms patients present with slight cyanosis, tachypnoea, and hepatomegaly with a moderately loud systolic murmur at the lower left sternal border. The chest $x$ ray shows pronounced cardiomegaly. In these patients the signs of tricuspid incompetence usually resolve within days, as they did in two of our patients ( 1 and 2). In severe forms (as in patient 3) postnatal distress and congestive heart failure may be life threatening. The electrocardiogram often shows evidence of myocardial ischaemia. ${ }^{4}$ Tricuspid regurgitation may be so massive that it impedes opening of the pulmonary valve. Pulmonary blood flow is then completely dependent on the ductus. Considerable shunting of systemic venous blood across the foramen ovale will result in pronounced desaturation of arterial oxygen. ${ }^{35}$ This conduction is known as "functional pulmonary atresia". ${ }^{12}$

The main difficulty in diagnosis is distinguishing between patients with organic pulmonary atresia, a dilated right ventricle, and tricuspid regurgitation" and those with isolated gross tricuspid regurgitation and "functional pulmonary atresia". 51213 The clinical, electrocardiographic, and radiographic findings are similar in these two groups. Cardiac catheterisation may be helpful in excluding pulmonary atresia if the catheter can be advanced into the pulmonary artery. In most cases of severe tricuspid regurgitation, however, this was difficult or impossible. ${ }^{512}$ If right ventricular systolic pressures are higher than systemic values there is a strong likelihood of severe obstruction of the right ventricular outflow and of secondary tricuspid regurgitation. But this anomaly may also be associated with only slightly raised or normal right ventricular pressures. ${ }^{8}$ On the other hand, right ventricular pressure in isolated tricuspid 
regurgitation varied from ${ }^{10 w^{36}}$ to considerably raised. ${ }^{5812}$ Right ventricular angiocardiography can be equally misleading. In patients with massive tricuspid regurgitation forward flow and passage of contrast material into the pulmonary artery may be minimal or absent, suggesting severe obstruction of the right ventricular outflow tract. ${ }^{5812}$ Freedom et al emphasised the role of aortography which they found was the only reliable method of distinguishing between functional and structural pulmonary atresia in 33 neonates with various forms of tricuspid regurgitation. ${ }^{12}$ In neonates with functional pulmonary atresia aortography showed a normal aortic isthmus (an indication of normal fetal ductal flow), a widely patent ductus arteriosus, a well developed pulmonary trunk, and, without exception, considerable regurgitation of contrast material into the right ventricle.

The clinical distinction between organic and functional pulmonary atresia is of considerable interest because management of the two conditions is different. Exploratory thoracotomy, in an attempt to relieve suspected obstruction of the right ventricular outflow tract, carries a high risk, ${ }^{58}$ whereas supportive medical treatment, including the administration of oxygen, correction of metabolic abnormalities, and administration of prostaglandins, may rapidly improve haemodynamic function and give time for spontaneous resolution of transient forms of tricuspid regurgitation in the newborn. ${ }^{356} \mathrm{We}$ too found that in our patients all signs of tricuspid incompetence resolved within the first days (two patients) or months of life (one patient).

Our study showed that Doppler echocardiography was useful in the evaluation and non-invasive follow up of haemodynamically compromised neonates presenting with tricuspid regurgitation. In all three cases echocardiography showed a structurally normal heart. Gross anomalies of the tricuspid valve, such as Ebstein's disease or severe dysplasia, could be excluded. In the third patient only, the tricuspid valve seemed to be thickened at the first examination, but the insertion of the annulus and the valve motion were normal. We noted changes that were secondary to gross tricuspid regurgitation: both the right atrium and ventricle were considerably dilated and the interatrial septum bowed towards the left atrium throughout the cardiac cycle, suggesting an increase in right atrial pressure. The ratio between the right ventricular and left ventricular end diastolic dimensions measured by $\mathbf{M}$ mode echocardiograms was much higher than the normal mean value $(0.60$ $(0.05))$ for neonates. ${ }^{13}$ The apex of the heart seemed to consist mainly of the right ventricle, even when the transducer was moved as far as possible to the left. In two patients movement of the pulmonary valve was normal or there was premature closure. It looked thickened and immobile in the patient with functional pulmonary atresia. A ductus arteriosus could be visualised in two of the three patients.

Doppler investigation showed moderate to massive tricuspid regurgitation, a right to left shunt through the foramen ovale, normal or reduced flow through the pulmonary valve, and, in the two cases in whom a ductus was visualised, ductal flow into the pulmonary artery. The findings in one patient with massive tricuspid regurgitation and functional pulmonary atresia are of special interest. The characteristic flow pattern of pulmonary regurgitation, which Freedom et al ${ }^{12}$ showed by aortography, was also displayed by the Doppler signal. Similar Doppler findings were reported in two neonates with functional pulmonary atresia secondary to severe Ebstein's anomaly. ${ }^{14}$ Imaging with colour Doppler will probably help to identify pulmonary regurgitation and distinguish between functional pulmonary atresia and organic pulmonary atresia.

Current continuous wave and pulsed Doppler methods are so sensitive that they commonly find regurgitant tricuspid flow patterns in normal individuals. ${ }^{75}$ Localised retrograde systolic flow, just proximal to the septal leaflets of the tricuspid valve, was detected in more than half of 11 healthy neonates with no clinically apparent tricuspid incompetence examined during the first days of life. ${ }^{10}$ In order to distinguish between these trivial forms of regurgitant flow and haemodynamically important tricuspid regurgitation we tsed several semiquantitative criteria for the area and the duration of retrograde turbulence. ${ }^{7}$ In two patients turbulent flow extended halfway into the right atrium and in the remaining patient it was recorded in the entire right atrium and in the hepatic veins. Turbulent flow was pansystolic whereas in normal neonates it was rarely found in more than half of systole. ${ }^{10}$ Furthermore, the Doppler findings should be interpreted in conjunction with the echocardiographic signs of tricuspid regurgitation, particularly gross dilatation of the right ventricle and right atrium, which were not seen in normal neonates. ${ }^{1013}$

\section{References}

1 Schiebler GL, Van Mierop LH, Krovetz LJ. Diseases of the tricuspid valve. In: Moss AJ, Adams FH, eds. Heart diseases in infants, children and adolescents. Baltimore: Williams and Wilkins, 1968:510.

2 Freymann R, Kallfelz HC. Transient tricuspid incompetence in a newborn. Eur $J$ Cardiol 1975;2:467-71.

3 Boucek RJ, Graham TJ, Morgan JP, Atwood GF, Boerth RC. Spontaneous resolution of massive congenital tricuspid insufficency. Circulation 1976; 54:795-800. 
4 Bucciarelli RL, Nelson RM, Egan EA, Eitzman DV, Gessner IH. Transient tricuspid insufficiency of the newborn: a form of myocardial dysfunction in stressed newborns. Pediatrics 1977;59:330-7.

5 Berman W, Whitman V, Stanger P, Rudolph AM. Congenital tricuspid incompetence simulating pulmonary atresia with intact ventricular septum. A report of two cases. Am Heart J 1978;96:655-61.

6 Berry TE, Muster AJ, Paul MH. Transient neonatal tricuspid regurgitation: possible relation with premature closure of the ductus arteriosus. J Am Coll Cardiol 1983;2:1178-82.

7 Miyatake K, Okamoto M, Kinoshita N, et al. Evaluation of tricuspid regurgitation by pulsed Doppler and two-dimensional echocardiography. Circulation 1982;66:777-84.

8 Barr PA, Celermajer JM, Bowdler JD, Cartmill TB. Severe congenital tricuspid incompetence in the neonate. Circulation 1974;49:962-7.

9 Davignon AL, Greenwold WE, Dushane JW, Edwards JE. Congenital pulmonary atresia with intact ventricular septum. Clinicopathologic correlation of two anatomic types. Am Heart J 1961;62:591-602.
10 Mahoney LT, Coryell KG, Lauer RM. The newborn transitional circulation: a two-dimensional Doppler echocardiographic study. J Am Coll Cardiol 1985; 6:623-9.

11 Rowe RD, Hoffman T. Transient myocardial ischemia of the newborn infant. A form of severe cardiorespiratory distress in full-term infants. $J$ Pediatr 1972;81:243-50.

12 Freedom RM, Culham G, Moes F, Olley PM, Rowe RD. Differentiation of functional and structural pulmonary atresia: role of aortography. Am J Cardiol 1978;41:914-20.

13 Azancot A, Caudell TP, Allen HD, et al. Analysis of ventricular shape by echocardiography in normal fetuses, newborns and infants. Circulation 1983;68:1201-11.

14 Smallhorn JF, Izukawa T, Benson L, Freedom RM. Noninvasive recognition of functional pulmonary atresia by echocardiography. Am J Cardiol 1984; 54:925-6.

15 Kostucki W, Vandenbossche JL, Friart L, Englert M. Pulsed Doppler regurgitant flow patterns in normal valves. Am J Cardiol 1986;58:309-14. 